\title{
Use of silicon for skin and hair care: an approach of chemical forms available and efficacy*
}

\author{
Lidiane Advincula de Araújo ${ }^{1}$ \\ Patrícia Maria Berardo Gonçalves Maia Campos ${ }^{1}$
}

Flavia Addor ${ }^{2}$

DOI: http:/ /dx.doi.org/10.1590/abd1806-4841.20163986

\begin{abstract}
Silicon is the second most abundant element on Earth, and the third most abundant trace element in human body. It is present in water, plant and animal sources. On the skin, it is suggested that silicon is important for optimal collagen synthesis and activation of hydroxylating enzymes, improving skin strength and elasticity. Regarding hair benefits, it was suggested that a higher silicon content in the hair results in a lower rate of hair loss and increased brightness. For these beneficial effects, there is growing interest in scientific studies evaluating the efficacy and safety of using dietary supplements containing silicon. Its use aims at increasing blood levels of this element and improving the skin and its annexes appearance. There are different forms of silicon supplements available and the most important consideration to be made in order to select the best option is related to safety and bioavailability. Silicon supplements are widely used, though there is wide variation in silicon bioavailability, ranging from values below $1 \%$ up to values close to $50 \%$, depending on the chemical form. Therefore, the aim of this study was to evaluate the scientific literature related to the different chemical forms of silicon supplements available and the limitations and recent progress in this field. According to reported studies, among the different chemical forms available, the orthosilicic acid (OSA) presents the higher bioavailability, whereas the others forms have absorption inversely proportional to the degree of polymerization. However, clinical studies evaluating safety and efficacy are still lacking.
\end{abstract}

Keywords: Biological availability; Collagen; Dietary supplements; Hair; Silicon; Silicon compounds; Skin aging

\section{INTRODUCTION}

Silicon is the second most abundant element on earth, exceeded only by oxygen. Also, it is the third most abundant trace element in the human body. ${ }^{1,2}$ It is present in the water and in plant and animal sources. On the skin, it is suggested that silicon is important for optimal synthesis of collagen and for activating the hydroxylation enzymes, improving skin strength and elasticity. It was shown that physiological concentrations of orthosilicic acid (OSA) stimulate fibroblasts to secrete collagen type $\mathrm{I}^{3-5}$ In the case of hair, it is suggested that higher silicon content in the hair fiber results in a lower rate of hair loss and increased brightness. Nails are also affected by the presence of silicon, since this is the predominant mineral in their composition. ${ }^{4,5}$ For these beneficial effects, there is growing interest in scientific studies to examine the efficacy and safety of the use of dietary supplements containing silicon, which aims to increase serum levels of this element and hence lead to improvements in the skin and its annexes. There are different forms of silicon supplements available and to select the most suitable option, the most important considerations to be made are regarding safety and bioavailability. In some countries, these supplements are already widely used, although there is great variation in silicon bioavailability, ranging from less than $1 \%$ up to values close to $50 \%$, depending on the chemical form.., 7

However, it is observed that there is still no consensus among researchers about the statement that silicon is an essential element for man or about the real benefits obtained from the use of supplements containing silicon. Thus, it is extremely important to critically evaluate the information published so far regarding efficacy, safety and bioavailability of silicon used in complementary supplements to the diet. That was the aim of this study. 


\section{SKIN AGING PROCESS}

The aging process occurs by two main mechanisms: intrinsic and extrinsic. The intrinsic aging is unavoidable and results in atrophy, fibroblasts reduction and thinning of blood vessels. The collagen fibers are particularly affected in this process, which results from the accumulation of irreversible degenerative changes associated with aging. 4,8,9 The extrinsic aging primarily results from damage caused by ultraviolet radiation. Other factors related to this type of aging include smoking, pollution and inadequate nutrition. These types of injury lead to increased degradation of collagen and elastin. Also, a reduction in the number of extracellular matrix proteins and a decrease in fibroblasts are described, ${ }^{8,9}$ in addition to a reduction of silicon levels and hyaluronic acid in the connective tissues. ${ }^{10}$

Collagen and fibers formed by it are responsible for the biomechanical properties of the skin, allowing it to act as an organ of protection from external trauma. They present as essential components of structural integrity of the connective tissue and are present in large quantities in the skin, bones and joints. ${ }^{9,11} \mathrm{~A}$ reduction in the amount of collagen in the skin of about $1 \%$ per year after 21 years of age is described, resulting in thickness reduction and elasticity loss, which is directly related to the wrinkles depth. ${ }^{11,12}$

Changes occurring after menopause are even more striking, including loss of about 30\% of skin collagen in the first 5 years and annual loss of $0.55 \%$ of elastin. ${ }^{13,14}$ The biosynthesis process of collagen after the third or fourth decade of life remains at a low level, insufficient to allow mature skin to repair or replace the collagen that has been lost as part of the degradation processes associated with age. ${ }^{9}$ The decrease of collagen that occurs after menopause especially correlates with decreased bone mineral density associated with age. ${ }^{14}$

By the study of skin aging process, it's possible to observe that the degradation of collagen fibers has a remarkable role in this context. Based on this, the use of mechanisms that influence the biosynthesis of this protein is as a potential tool for improving and preventing skin aging.

\section{SILICON: A UBIQUITOUS ELEMENT}

Considering the abundance of silicon in the human body, it seems unlikely that its deficiency occurs in men and women. ${ }^{15}$

In 1972, two studies by two different research groups showed that silicon was an essential element in chickens and mice. ${ }^{16,17}$ These experiments demonstrated that nutritional deficiencies of silicon led to skeletal deformities such as abnormal skull and long bone structures, as well as malformed joints with cartilage poor content. Thus, an important role of silicon in bone mineralization was demonstrated.
After that, several studies showed silicon participation in different mechanisms, with positive results associated with higher concentrations of this element in the blood in patients with osteoporosis, atherosclerosis, skin aging and fragile hair and nails. ${ }^{10,15}$ However, there are no conclusive data to determine whether or not silicon is an essential nutrient for humans and superior animals, since its deficiency has not led to cell cycle interruption in mammals, and its functional role remains to be clearly defined. ${ }^{15,18-20}$ Most of the silicon present in the blood is filtered by the kidneys, suggesting that this mechanism represent the major route of excretion and that levels of silicon in blood correlate with the levels present in urine..$^{21}$ For this reason, various studies evaluate the serum concentration as well as the one present in urine in order to study the bioavailability of silicon and its derivatives.

Silicon occurs naturally in foods in the form of silicon oxide and silicates, which are present in water and in plant and animal sources and are found in high concentrations especially in cereals. ${ }^{18,22}$ The main sources of silicon from the diet in the Western Hemisphere are cereals $(30 \%)$, followed by fruit, beverage and vegetable-derived products in general. Together, these foods provide about $75 \%$ of the total silicon ingested by man. ${ }^{23}$

However, there are studies that question the bioavailability of silicon from some sources, due to the low solubility of some compounds, especially those that are polymerized. ${ }^{15,24}$ Thus, although significant quantities of silicon are present in some foods, sometimes it is presented in an insoluble form and cannot be directly absorbed in the gastrointestinal tract. The silicon present in food is solubilized in the acid environment of stomach, becoming OSA [Si $(\mathrm{OH}) 4$ ], which can then be absorbed. It is described in the literature that the aging process is associated with an increase in gastric $\mathrm{pH}$, which decreases the conversion capacity of this silicon found in foods in the bioavailable form. ${ }^{5}$

OSA is the main type derived from silicon present in drinking water and other liquids, including beer, and it is considered the most readily available form of silicon to humans. ${ }^{19}$ It is stable when diluted $\left(<10^{-4} \mathrm{M}\right)$ but polymerizes in higher concentrations in a $\mathrm{pH}$ close to neutral. Absorption studies indicated that only OSA is available while its polymerized form is not absorbed. ${ }^{25}$ Questions on the bioavailability of silicon from the mineral water are reported in the literature. In a study conducted with rats that received supplementation with OSA in the water they ingested, there were no significant differences in the concentration of silicon present in bones in relation to baseline. ${ }^{1}$ In beer, it demonstrated that about $80 \%$ of the total silicon found corresponds to OSA. ${ }^{26}$ However, there are discussions involving the availability of OSA, which could be unstable in industrial processes such as, for example, bottling. 
At high concentrations, OSA needs to be stabilized so it doesn't polymerize excessively, resulting in a reduced bioavailability. ${ }^{10}$ For this reason, silicon-containing supplements attempt, by different methods, to concentrate OSA and stabilize it in a way to make it more bioavailable.

\section{FOOD SUPPLEMENTS CONTAINING SILI-} CON

Different consumptions patterns of supplements containing silicon are observed around the world. As an example, the organic silicon - commonly the monomethyl silanetriol (MMST) - is more consumed in France, while in Germany the colloidal silicon are more present and, in Belgium, choline-stabilized OSA (ch-OSA) is more frequent. ${ }^{6,7}$

The MMST is not only organic, but also monomeric while other silicates show different degrees of polymerization, which should explain the different silicon absorption values in experiments with rats and in some preliminary studies in humans. ${ }^{25,27}$ Some studies have shown that it is readily absorbed after digestion and observed no adverse events with its use. Nevertheless, it is noteworthy that, until the completion of these works, specific studies to evaluate its safety were not conducted..$^{28}$

Jugdaohsingh et al, in 2013, conducted a study to assess the safety of using this supplement. A group of 22 healthy women, who were not menopausal, received MMST oral supplementation for 4 weeks, with the maximum recommended dose of $10.5 \mathrm{mg} / \mathrm{Si} /$ day. The authors concluded that MMST intake is safe and that it was absorbed. They also presented data to prove that, after ingestion, there is conversion of MMSR in OSA, which would justify its absorption..$^{28}$

However, in response to the published article, Vanden Berghe questioned some points of the study, claiming that studies of longer duration in humans and toxicological tests in vitro and in animals are needed in order to prove the safety of using the supplement containing MMST. According to Vanden Berghe, these studies were not presented in the article in question and they are also scarce in the available literature on the subject. The statement on MMST conversion in OSA was also questioned..$^{29}$

The authors of the original study published a response that kept emphasizing the study's findings. They argued that they used rigorous methodology and that, in the adopted conditions, they could conclude it was safe to use the supplement containing MMST. The authors, however, agreed that studies with larger numbers of volunteers and greater length of time would be needed for the continuation of research involving this supplement. ${ }^{30}$

MMST has been used as a silicon source for a long time around the world, especially in Europe. ${ }^{28}$ This supplement, unlike others available, does not contain nano-silica particles, on which concerns regarding the safety have been reported. ${ }^{31,32}$ However, the European Food Safety Authority (EFSA) considers that there is not enough data to justify the use of MMST as silicon supplement. ${ }^{19}$

The greatest number of studies in the literature evaluates ch-OSA. The ch-OSA has been approved for human consumption and is known to be non-toxic, in addition to representing the most bioavailable form of silicon. . $^{15,22}$

In chemical terms, ch-OSA is a mixture of OSA and choline chloride. Given the lack of data about adverse reactions to silicon, a recommended dose has not been established. Nevertheless, according to the American regulatory agency, choline, silicon oxides and various silicates are classified as substances "generally recognized as safe" ${ }^{18,19}$

The stabilization with choline is considered the most advanced technology for OSA stabilization. Choline has important characteristics that place it in the position of an ideal stabilizer for OSA, in addition to promoting benefits due to its own characteristics. ${ }^{33} \mathrm{In}$ high concentrations, choline avoids extensive polymerization and aggregation of silicon particles, to keep it in an aqueous suspension. ${ }^{33}$

Furthermore, as previously mentioned, choline present in the compound may have a synergistic effect with OSA, since it is well known its participation in many basic biological processes. ${ }^{33}$ Choline is a precursor of phospholipids, which are essential for the formation of cell membranes, as well as being involved in processes such as cell signaling, lipid metabolism and protection against the collagen breakdown mediated by homocysteine. ${ }^{34,35}$

In 2009, EFSA requested a scientific opinion to the Panel on Food Additives and Nutrient Sources Added to Food concerning ch-OSA safety. The only objective was to evaluate ch-OSA as a silicon source and also its bioavailability. Thus, silicon safety itself, in terms of daily amounts that can be consumed and its classification as a nutrient, was outside the scope of scientific opinion published by the Panel. ${ }^{19}$

Based on different studies conducted in animals and in humans, the Panel concluded that the silicon present in ch-OSA is bioavailable and that its use in supplements, in the proposed doses, does not present risks for safety, providing that the choline maximum level is not exceeded ( $3.5 \mathrm{~g} /$ day).

Studies were analyzed both in animals and in humans so the conclusion on bioavailability and safety were published by EFSA. A study of calves that received supplement containing ch-OSA or placebo for 23 weeks evaluated the evolution of serum silicon con- 
centration. There was a $4.9 \%$ increase at this concentration in the group of animals receiving silicon. ${ }^{36}$ In another study, Vanden Berghe assessed the bioavailability of silicon in offspring of 21 pigs, which received or not (control) supplement containing ch-OSA during the gestation (16 weeks) and lactation (four weeks) period. In the offspring of pigs that received supplement containing silicon, significantly higher silicon concentrations were found ( $150 \%$ increase) than in the offspring of the control group. The authors attributed this result to the bioavailability of silicon in the supplement containing ch-OSA and also to the maternal transfer capability of absorbed silicon. ${ }^{37}$ The silicon absorption from supplement containing ch-OSA was assessed in a study of 14 healthy volunteers aged 22-34 years. Each volunteer received successive oral doses of silicon from different sources. A significant increase in serum concentration of silica compared to baseline was observed for ch-OSA. ${ }^{6,19}$ This study demonstrated that the bioavailability of silicon is to a great extent dependent on the chemical form of the compound.

In another study, conducted in order to examine in vivo absorption of silicon by evaluating its serum dosage and its urinary excretion, different patterns of absorption for the different sources used were found. This study obtained different results, depending on the source, although it has evaluated absorption in just a healthy volunteer. It was observed that a diet rich in silicon does not result in sufficient bioavailable amounts of this element that would lead to a statistically significant increase in its urinary excretion and serum levels, when compared with the period in which the volunteer was subjected to a normal diet. A significant increase in silicon urinary excretion was observed when the evaluated supplementation consisted of tablets containing dry extract of horsetail. However, the silicon serum levels remained constant. Only the biologically active silicon present in solution at $2 \%$ silicon in a matrix of choline and glycerol was absorbed, which reflected in the significant increase of silicon in both serum levels and in urine excretion. Based on this study, the authors concluded that silicon absorption is strongly influenced by its chemical form and matrix.

Sripanyakorn et al measured silicon uptake from 8 different sources. In healthy volunteers, blood and urine samples were analyzed to quantify the concentration of silicon. The results confirmed that the degree of silicon polymerization is inversely proportional to intestinal absorption. ${ }^{7}$

\section{SILICON AND ITS RELATION WITH SKIN, HAIR AND NAILS}

Regarding the skin, it is suggested that silicon is important for optimal synthesis of collagen and for activating the hydroxylation enzymes, important in the formation of collagen network, improving skin strength and elasticity. Silicon is also associated with the synthesis of glycosaminoglycans. Concerning the hair, it is suggested that strands with higher silicon content tend to have lower falling rate and higher brightness. Nails are also affected by the presence of silicon, since this element is one of the predominant mineral in their composition. The presence of soft and brittle nails can indicate systemic deficiency of silicon. By improving the quality of nails, there is an increased protection against nail infections. ${ }^{4,5}$

In a study with 50 healthy volunteers, aged between 40 and 65 years and with clear clinical signs of facial photoaging, the effect of the intake of supplements containing ch-OSA to the skin, hair and nails were analyzed. The supplement was held for a period of 20 weeks, with 2 capsules containing $10 \mathrm{mg}$ of chOSA taken daily. Also, serum concentrations of various components in the blood were evaluated in order to verify safety of oral treatment. The silicon intake under these experimental conditions was considered safe, since there were no reported adverse events with this treatment. This study, according to the authors, was the first randomized, double-blind, placebo-controlled trial that showed positive results in the skin microtopography and anisotropy after the intake of supplement containing ch-OSA. At the end of the period of use supplement containing silicon, there was a significant improvement in the skin surface characteristics and in its mechanical properties.

Also in this study, it was observed a significant improvement in the fragility of nails and hair in the group using the ch-OSA. The placebo did not lead to significant differences in rating assigned by the volunteers by the self-assessment questionnaires completed before the start and after the end of the study.

Another randomized study with 48 volunteers investigated the effect of ch-OSA on hair. The volunteers had thin hairs and were divided into 2 groups: ch-OSA and placebo. The first group received daily doses of $10 \mathrm{mg}$ of silicon, for a period of 9 months. ${ }^{4}$ Morphology and mechanical properties of hair were evaluated at the beginning and at the end of the study. In general, positive results were obtained in the evaluated hair properties, such as strand resistance to breaking, for example. Furthermore, the area of the strand front section increased significantly after 9 months of supplementation containing ch-OSA, whereas the placebo group exhibited no significant difference. ${ }^{4}$

The fact that ch-OSA have partially prevented the loss of hair tensile strength suggests that it has a structural effect on hair fibers. According to the authors, an interaction with keratin is possible, considering that OSA is the chemical form of silicon prevalent in physiological fluids and that silanol group, present on OSA, is known to form complexes with amino acids and peptides. ${ }^{4,38,39}$ 


\section{CONCLUSION}

The analysis of the scientific literature on the use of supplements containing silicon shows great therapeutic potential of this element, as it operates in different conditions of human health and presents aesthetic properties. Among the various chemical forms available, the analysis of studies shows that OSA is the form that presents greater bioavailability; other forms have absorption inversely proportional to the degree of polymerization. We also observed that ch-OSA is the most referenced form in the literature, suggesting a greater scientific support regarding its use. However, there are few studies evaluating the safety, efficacy and bioavailability of the different existing chemical forms of silicon that use proper design, large number of volunteers and long follow-up period.]

\section{REFERENCES}

1. Jugdaohsingh R, Calomme MR, Robinson K, Nielsen F, Anderson SH, D'Haese $P$, et al. Increased longitudinal growth in rats on a silicon-depleted diet. Bone. 2008;43:596-606.

2. Reffitt DM, Jugdaohsingh R, Thompson RP, Powell JJ. Silicic acid: its gastrointestinal uptake and urinary excretion in man and effects on aluminium excretion. J J Inorg Biochem. 1999;76:141-7.

3. Reffitt DM, Ogston N, Jugdaohsingh R, Cheung HF, Evans BA, Thompson RP, et al. Orthosilicic acid stimulates collagen type 1 synthesis and osteoblastic differentiation in human osteoblast-like cells in vitro. Bone. 2003;32:127-35.

4. Wickett RR, Kossmann E, Barel A, Demeester N, Clarys P, Vanden Berghe D, et al. Effect of oral intake of choline-stabilized orthosilicic acid on hair tensile strength and morphology in women with fine hair. Arch Dermatol Res. 2007;299:499-505.

5. Lef.org [Internet]. Silicon: An Overlooked Trace Mineral Silicon. 2003 p. 1-6. [Cited 2003 apr 1]. Available from: http://www.lef.org/

6. Calomme MR, D'Haese PC, Vingerhoets R, Lamberts LV, De Broe ME, Van Hoorebeke DA, et al. Absorption of silicon in healthy subjects. In: Collery P, Brätter P, Negretti De Brätter V, Khassanova L, Etienne JC, editors. Metal lons in Biology and Medicine. vol V. Paris: John Libbey Euro Text; 1998. p. 228-32.

7. Sripanyakorn S, Jugdaohsingh R, Dissayabutr W, Anderson SH, Thompson RP, Powell JJ. The comparative absorption of silicon from different foods and food supplements. Br J Nutr. 2009; 102:825-34.

8. Fanian F, Mac-Mary S, Jeudy A, Lihoreau T, Messikh R, Ortonne JP, et al. Efficacy of micronutrient supplementation on skin aging and seasonal variation: a randomized, placebo-controlled, double-blind study. Clin Interv Aging. 2013;8:1527-37.

9. Jouni Uitto MD. The role of Elastin and Collagen in Cutaneous Aging: Intrinsec Aging Versus Photoexposure. J Drugs Dermatol. 2008;7:s12-6.

10. Jurkić LM, Cepanec I, Pavelić SK, Pavelić K. Biological and therapeutic effects of ortho-silicic acid and some ortho-silicic acid-releasing compounds: New perspectives for therapy. Nutr Metab (Lond). 2013;10:2.

11. Shuster S. Osteoporosis, a unitary hypothesis of collagen loss in skin and bone. Med Hypotheses. 2005;65:426-32.

12. Akazaki S, Nakagawa $\mathrm{H}$, Kazama H, Osanai 0 , Kawai M, Takema $\mathrm{Y}$, et al. Clinical and Laboratory Investigations Age-related changes in skin wrinkles assessed by a novel three-dimensional morphometric analysis. Br J Dermatol. 2002;147:689-95.

13. Baumann L. Skin ageing and its treatment. J Pathol. 2007;211:241-51.

14. Sumino H, Ichikawa S, Abe M, Endo Y, Ishikawa O, Kurabayashi M. Effects of aging, menopause, and hormone replacement therapy on forearm skin elasticity in women. J Am Geriatr Soc. 2004;52:945-9.

15. Van Dyck K, Van Cauwenbergh $R$, Robberecht $H$, Deelstra $H$. Bioavailability of silicon from food and food supplements. Fresenius J Anal Chem. 1999; 363:541-4.

16. Carlisle ME. Silicon: an Essential Element for the Chick. Science. 1972; 178:619-21.

17. Schwarz K, Milne DB. Growth-promoting Effects of Silicon in Rats. Nature. 1972; 239:333-4.

18. Fda.gov [Internet]. Dioxides, Select Committee on GRAS Substances (SCOGS) Opinion: Silicon. Center for Food Safety and Applied Nutrition; 2013 [cited 2014 Jul 21]. Available from: http://www.fda.gov/food/ingredientspackaginglabeling/ gras/scogs/ucm261095.htm

19. Aguilar F, Charrondiere UR, Dusemund B, Galtier P, Gilbert J, Gott DM, et al. Scientific Opinion. Choline-stabilised orthosilicic acid added for nutritional purposes to food supplements. Scientific Opinion of the Panel on Food Additives and Nutrient Sources added to Food. EFSA J. 2009; 948:1-23.

20. Nielsen FH. Update on the possible nutritional importance of silicon. J Trace Elem Med Biol. 2014; 28:379-82.

21. Berlyne GM, Adler AJ, Ferran N, Bennett S, Holt J. Silicon metabolism. I. Some aspects of renal silicon handling in normal man. Nephron. 1986;43:5-9.
22. Opinion of the Scientific Panel on Dietetic Products, Nutrition and Allergies on a request from the Commission related to the Tolerable Upper Intake Level of Silicon. EFSA J. 2004;60: 1-11

23. McNaughton SA, Bolton-Smith C, Mishra GD, Jugdaohsingh R, Powell JJ. Dietary silicon intake in post-menopausal women. Br J Nutr. 2005; 94:813-7.

24. Bellia JP, Birchall JD, Roberts NB. Beer: a dietary source of silicon. Lancet. 1994 343:235.

25. Jugdaohsingh R, Reffitt DM, Oldham C, Day JP, Fifield LK, Thompson RP, et al. Oligomeric but not monomeric silica prevents aluminum absorption in humans. Am J Clin Nutr. 2000; 71:944-9.

26. Sripanyakorn S, Jugdaohsingh R, Elliott H, Walker C, Mehta P, Shoukru S, et al The silicon content of beer and its bioavailability in healthy volunteers. $\mathrm{Br} \mathrm{J}$ Nutr. 2004;91:403-9.

27. Jugdaohsingh R, Anderson SH, Tucker KL, Elliott H, Kiel DP, Thompson RP, et al. Dietary silicon intake and absorption 1 - 3. Am J Clin Nutr. 2002; 75:887-93.

28. Jugdaohsingh $\mathrm{R}$, Hui M, Anderson SH, Kinrade SD, Powell JJ. The silicon supplement "Monomethylsilanetriol "is safe and increases the body pool of silicon in healthy Pre-menopausal women. Nutr Metab (Lond). 2013;10:37.

29. Vanden Berghe DA. There are not enough data to conclude that Monomethylsilanetriol is safe. Nutr Metab (Lond). 2013; 10:66

30. Jugdaohsingh R, Anderson SH, Kinrade SD, Powell JJ. Response to Prof D . Vanden Berghe letter: There are not enough data to conclude that Monomethylsilanetriol is safe. Nutr Metab (Lond). 2013; 10:65.

31. Gehrke H, Frühmesser A, Pelka J, Esselen M, Hecht LL, Blank H, et al. In vitro toxicity of amorphous silica nanoparticles in human colon carcinoma cells. Nanotoxicology. 2013; 7:274-93.

32. Napierska D, Thomassen LC, Lison D, Martens JA, Hoet PH. The nanosilica hazard: another variable entity. Part. Fibre Toxicol. Part Fibre Toxicol. 2010;7:39.

33. Bio Minerals NV. Ch-OSA (choline-stabilized orthosilicic acid). Clinically Proven. ch-OSA monograph 2007. p. 1-11.

34. Zeisel SH, da Costa KA. Choline: an essential nutrient for public health. Nutr Rev. 2009; 67:615-23.

35. Blusztajn JK. Choline, a Vital Amine. Science. 1998;281:794-5

36. Calomme MR, Vanden Berghe DA. Supplementation of Calves with Stabilized Orthosilicic Acid Effect on the $\mathrm{Si}, \mathrm{Ca}, \mathrm{Mg}$, and P Concentrations in Serum and the Collagen Concentration in Skin and Cartilage. Biol Trace Elem Res. 1997; 56:153-65.

37. Vanden Berghe DA. Supplementation of sows and rats with ch-OSA, a safety assessment. Belgium; 2004.

38. Coradin T, Livage J. Effect of some amino acids and peptides on silicic acid polymerization. Colloids Surf B Biointerfaces. 2001; 21:329-336.

39. Coradin T, Lopez PJ. Biogenic silica patterning: simple chemistry or subtle biology? Chembiochem. 2003;4:251-9.

MAILING ADDRESS:

Patrícia Maria Berardo Gonçalves Maia Campos

Avenida do Café, S/N

Monte Alegre

14040-903 - Ribeirão Preto - SP - Brazil

Email:pmcampos@usp.br

How to cite this article: Araújo LA, Addor F, Campos PMBGM. Use of Silicon for skin and hair care: an approach of chemical forms available and efficacy. An Bras Dermatol. 2016;91(3):331-5. 